\section{Is incidental recovery of yeast from enteric pathogen stool cultures obtained from hospitalized patients clinically significant?}

\author{
Rachel A. Pfeifer, ${ }^{1}$ Peter C. Iwen, ${ }^{2}$ \\ Fang Qiu, ${ }^{3}$ Mark E. Rupp, ${ }^{4}$ \\ Elizabeth D. Hermsen ${ }^{1,4,5}$ \\ ${ }^{1}$ The Nebraska Medical Center, \\ Department of Pharmaceutical \& \\ Nutrition Care, Omaha, NE; \\ 'University of Nebraska Medical Center, \\ College of Medicine, Department of \\ Pathology and Microbiology, Omaha, NE, \\ USA; ${ }^{3}$ University of Nebraska Medical \\ Center, College of Public Health, \\ Department of Biostatistics, Omaha, NE; \\ ${ }^{4}$ University of Nebraska Medical Center, \\ College of Medicine, Department \\ of Internal Medicine, Section of Infectious \\ Diseases, Omaha, NE; ${ }^{5}$ University of \\ Nebraska Medical Center, College \\ of Pharmacy, Department of Pharmacy \\ Practice, Omaha, NE, USA
}

\section{Abstract}

A matched case-control study was conducted to investigate gastrointestinal colonization with yeast as a predictor of invasive candidiasis (IC) in patients who underwent an enteric pathogen test. No significant association was detected between gastrointestinal colonization and IC. However, gastrointestinal colonization with yeast was associated with increased antimicrobial exposure and median length of hospitalization.

\section{Introduction}

Invasive candidiasis (IC) is a serious infection associated with a high mortality rate and limited definitive diagnostic signs..$^{1-3}$ Coloniza tion is a key component of IC pathogenesis, yet the significance of gastrointestinal Candida colonization as a predictor of IC remains controversial. ${ }^{3-5}$

Although yeast are normal bowel flora and recovery of low numbers in stool cultures is common, some hospitalized patients exhibit large numbers of yeast in stools obtained for enteric pathogen screening. The clinical significance of this finding is not well defined. Hospitalized patients undergoing evaluation for a possible inflammatory etiology of diar- rhea may heighten concern that heavy yeast colonization of the gastrointestinal tract could progress to IC. Clinicians question whether prophylaxis of IC is warranted in such cases, and clinical microbiologists question whether the reporting of heavy yeast carriage is necessary. The study objective was to determine if a relationship exists between IC and recovery of large numbers of yeast from stool cultured for enteric pathogens.

All inpatients at a 689-bed academic medical center from January 2004-July 2008 with an enteric pathogen stool culture revealing yeast were eligible for inclusion. Only stools with moderate to many yeast detected (growth in two or more quadrants of an isolation streak from the original stool on sheep blood agar) were evaluated. Routine surveillance cultures for gastrointestinal colonization were not performed. Sheep blood agar, incubated for 72 hours, was included as a primary medium to screen for Aeromonas hydrophila and recognize patients that lack stool flora. Each case patient, included only once, was matched by age, gender, and admission date in a 1:3 ratio to subjects with enteric pathogen stool cultures negative for yeast in a retrospective matched case-control study. Subjects with IC prior to the index stool and those who received systemic antifungals within 30 days after the index stool were excluded. The study received Institutional Review Board approval with a complete waiver of informed consent.

The primary outcome was development of IC, identified from an institutional database of patients diagnosed with IC and defined by a positive culture from blood, intra-abdominal abscess, peritoneal fluid, or cerebrospinal fluid indicative of IC. A subgroup analysis was planned for immunocompetent versus immunocompromised subjects.

A retrospective chart review was done to collect the following: demographics; admission/ discharge dates; culture/susceptibility results; presence and date of IC; antimicrobial, immunosuppressant, and chemotherapy regimens; mechanical ventilation; catheterizations (intravascular and urinary); hemodialysis; and parenteral nutrition received during hospitalization, as well as internal prosthetic devices and surgical procedures performed within 30 days prior to index stool.

Wilcoxon rank sum and Chi-square or Fisher's exact tests were used for continuous and categorical variables, respectively. P-values less than 0.05 were considered significant.

In total, 115 unique subjects had a stool culture that revealed moderate to many yeast out of 2373 total unique stool cultures performed among hospitalized patients during the study period. Fifty-seven subjects were excluded [developed IC prior to index stool $(n=1)$, received systemic antifungals after index stool $(\mathrm{n}=40)$, and outpatient status at time of cul-
Correspondence: Elizabeth D. Hermsen, 65 Hayden Avenue, Lexington, MA 02421, USA.

E-mail: elizabeth.hermsen@cubist.com

Key words: candidiasis, colonization, stool culture.

Acknowledgements: we are grateful to Dalal Gumeel, PharmD Candidate at the University of Nebraska Medical Center College of Pharmacy, for her assistance with data collection. Financial support: none.

Contributions: RAP contributed to the study design and execution and manuscript preparation; PCI contributed to the study concept and design, data acquisition, and manuscript preparation; FQ performed the statistical analysis and assisted with manuscript preparation; MER contributed to the study design and manuscript preparation; EDH contributed to the study concept and design, execution of the study, and manuscript preparation.

Conflict of interest: EDH is currently an employee of Cubist Pharmaceuticals, is a shareholder in Cubist Pharmaceuticals, has been a consultant for Ortho-McNeil Pharmaceuticals, Cubist Pharmaceuticals, and Forest Laboratories, and has received research funding from Pfizer and TheraDoc. MER has received research funding from Cubist Pharmaceuticals, Sanofi-Pasteur, Cardinal Health Foundation, 3M, and Molynlcke. All other authors report no conflicts of interest.

Received for publication: 7 December 2010. Revision received: 19 January 2011. Accepted for publication: 20 January 2011.

This work is licensed under a Creative Commons Attribution 3.0 License (by-nc 3.0).

(C) Copyright R.A. Pfeifer et al., 2011

Licensee PAGEPress, Italy

Infectious Disease Reports 2011; 3:e1

doi:10.4081/idr.2011.el

ture $(n=16)]$. The remaining cases $(n=58)$ were matched to 174 controls in a 1:3 ratio. Study subject characteristics are summarized in Table 1. No significant differences were detected between cases and controls for age, gender, and ethnicity, (data not shown) but antimicrobial use was significantly more common in cases versus controls (95\% vs. $73 \%$, $\mathrm{P}=0.0005$ ). The length of hospitalization was significantly longer (median, 2.5 days longer) for cases $(\mathrm{P}=0.002)$.

One control subject developed IC. No association was identified between IC and risk factors collected. No difference between the development of IC among immunocompetent versus immunocompromised patients was detected.

Other studies investigating candidal colonization and systemic disease have neither focused on incidental demonstration of heavy gastrointestinal colonization from an enteric 
pathogen culture nor firmly concluded a correlation between colonization and subsequent IC.

Magill et al. investigated whether colonization site impacted the likelihood of developing IC in 182 surgical intensive care unit (SICU) patients. ${ }^{5}$ No sites (urine, oropharynx, trachea, gastric aspirate, or rectum/ostomy) were associated with a high positive predictive value for IC. Conversely, all patients with negative rectum/ostomy, urine, and tracheal aspirate cultures remained without IC. The high negative predictive values for these cultures suggest a limited strategy to identify patients who are unlikely to benefit from antifungal prophylaxis. ${ }^{5}$

Blumberg et al. assessed risk factors for candidemia in a multicenter, prospective cohort involving SICU patients $(\mathrm{n}=4276){ }^{4}$ Recovery of Candida from rectum and urine samples was not associated with a significant risk of developing candidemia. The authors concluded urinary or rectal colonization alone did not predict patient populations for antifungal prophylaxis. ${ }^{4}$

While these findings, in addition to the present study, suggest that rectal/gastrointestinal colonization alone does not predict IC, another study suggested that gastrointestinal colonization was the source for $C$. albicans candidemia. ${ }^{6}$ However, surveillance cultures were obtained after candidemia documentation, and thus, whether gastrointestinal tract colonization preceded candidemia was not elucidated.

Other investigators have incorporated colonization as one of many risk factors to be included in rules to help predict whether a given patient is likely to develop IC. ${ }^{7-9}$ These studies have focused on patients at high risk for IC and have included colonization sites other than the gastrointestinal tract. Additionally, the ability to include colonization with yeast in such a clinical prediction rule requires routine surveillance cultures, which are not performed at our institution. These studies demonstrate that the predictive value of colonization for invasive candidiasis is variable.

In our study, the only case of IC occurred in a control subject who did not have heavy gastrointestinal colonization with yeast. Thus, if a negative gastrointestinal culture had been used to decide upon prophylactic therapy, this patient would not have received prophylaxis.

This matched case-control study was intended to investigate the significance of incidental demonstration of substantial gastrointestinal yeast colonization as a predictor of IC. Although conducted in a general population not selected as high risk for IC, many risk factors were present in both cases and controls (Table 1). Since cultures collected for detection of enteric pathogens were used, these observations may not be applicable to other situations. Furthermore, only patients who had an enteric stool specimen submitted for analysis were included in the study, and thus, these results may not be generalizable to the majority of patients who do not have stool specimens collected. Other limitations include the retrospective, single-centered study design, small sample size, and lack of data regarding inten-

Table 1. Patient characteristics comparing patients with gastrointestinal yeast colonization to those without colonization.

\begin{tabular}{|c|c|c|c|}
\hline \multirow[t]{2}{*}{ Patient characteristic } & \multicolumn{2}{|c|}{ Yeast in stool culture } & \multirow[t]{2}{*}{$\mathbf{P}$} \\
\hline & $\begin{array}{l}\text { Positive } \\
(\mathrm{n}=58) \\
n(\%)\end{array}$ & $\begin{array}{c}\text { Negative } \\
(n=174) \\
n(\%)\end{array}$ & \\
\hline Received antimicrobials ${ }^{\mathrm{a}}$ & $55(94.8)$ & $127(72.9)$ & 0.0005 \\
\hline Received immunosuppressants ${ }^{a}$ & $24(41.4)$ & $63(36.2)$ & 0.481 \\
\hline Received chemotherapya & $0(0)$ & $2(1.1)$ & 0.99 \\
\hline Medical prosthetic devices ${ }^{b}$ & $12(20.7)$ & $20(11.5)$ & 0.08 \\
\hline Surgical procedures ${ }^{\mathrm{b}}$ & $19(32.8)$ & $41(23.6)$ & 0.166 \\
\hline Mechanical ventilation $^{\mathrm{a}}$ & $11(19)$ & $20(11.5)$ & 0.148 \\
\hline Intravascular catheterization ${ }^{\mathrm{a}}$ & $44(75.9)$ & $113(65)$ & 0.12 \\
\hline Hemodialysis $^{\mathrm{a}}$ & $4(6.9)$ & $12(7.5)$ & 0.99 \\
\hline Parenteral nutrition ${ }^{\mathrm{a}}$ & $8(13.8)$ & $28(16.1)$ & 0.68 \\
\hline $\begin{array}{l}\text { Total length of hospitalization } \\
\text { [median (range)] }\end{array}$ & $8.5(1,116)$ & $6.0(1,231)$ & 0,002 \\
\hline
\end{tabular}

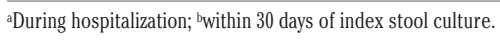

sive care unit days.

This study suggests an association between antimicrobial exposure and gastrointestinal colonization with yeast. Additionally, a significant difference was found in length of hospital stay between cases and controls, suggesting more time in the hospital may increase the probability of colonization. No significant correlation between IC and incidental yeast detection in stool cultures was observed.

\section{References}

1. Guery BP, Arendrup MC, Auzinger G, et al. Management of invasive candidiasis and candidemia in adult non-neutropenic intensive care unit patients: Part I. Epidemiology and diagnosis. Intensive Care Med 2009;35:55-62.

2. Horn DL, Neofytos D, Anaissie EJ, et al. Epidemiology and outcomes of candidemia in 2019 patients: data from the prospective antifungal therapy alliance registry. Clin Infect Dis 2009;48:1695-703.

3. Pappas PG. Invasive candidiasis. Infect Dis Clin North Am 2006;20:485-506.

4. Blumberg HM, Jarvis WR, Soucie JM, et al. Risk factors for candidal bloodstream infections in surgical intensive care unit patients: the NEMIS prospective multicenter study. The National Epidemiology of Mycosis Survey. Clin Infect Dis 2001;33: 177-86.

5. Magill SS, Swoboda SM, Johnson EA, et al. The association between anatomic site of Candida colonization, invasive candidiasis, and mortality in critically ill surgical patients. Diagn Microbiol Infect Dis 2006; 55:293-301.

6. Miranda LN, van der Heijden IM, Costa SF, et al. Candida colonisation as a source for candidaemia. J Hosp Infect 2009;72:9-16.

7. Leon C, Ruiz-Santana S, Saavedra P, et al. A bedside scoring system (Candida score) for early antifungal treatment in nonneutropenic critically ill patients with Candida colonization. Crit Care Med 2006;34:730-7.

8. Pittet D, Monod M, Suter PM, et al. Candida colonization and subsequent infections in critically ill surgical patients. Ann Surg 1994;220:751-8.

9. Playford EG, Lipman J, Kabir M, et al. Assessment of clinical risk predictive rules for invasive candidiasis in a prospective multicentre cohort of ICU patients. Intensive Care Med 2009;35:2141-5. 